\title{
Navigating the Needs of Rural Women with Breast Cancer: A Breast Care Program
}

\author{
Jill L. Depke, MSN, APNP, AOCNP, CBCN; Amanda Boreen, RN, CBPN-IC, CBCN; \\ and Adedayo A. Onitilo, MD, PhD, MSCR, FACP
}

\begin{abstract}
We describe the development and establishment of a breast care program (BCP) with service for rural breast cancer patients. Our program is a comprehensive program serving rural communities in Wisconsin. Our BCP is committed to breast health throughout the continuum from breast cancer risk assessment and prevention, advanced diagnostics, and screening tools to genetic testing and state-ofthe-art surgical techniques. To provide the highest level of care, we coordinate a breast care team involving collaboration of multidisciplinary healthcare professionals. Experts from various departments, including radiologists, pathologists, breast surgeons, medical and radiation oncologists, genetic counselors, clinical trial specialists, and our breast care navigator, all work together to provide cutting edge cancer treatment and management. Our distinctive $\mathrm{BCP}$ allows patients to see multiple providers without having to make multiple appointments and promotes discussion of treatment recommendations and creation of a personalized treatment plan for each patient by a team of specialists.
\end{abstract}

Keywords: Breast neoplasms/therapy; Health care delivery; Multidisciplinary healthcare;

Outcome and process assessment; Patient satisfaction; Referral and consultation

$\mathrm{B}$ United States.' Advances in the diagnosis and treathent of breast cancer have resulted in a gradual decline in mortality over the last 30 years, but this has also made the system of care complex and difficult to navigate. Breast care programs offer an effective solution to the navigation issue. The Marshfield Clinic Breast Care Program is a comprehensive breast care program serving rural Wisconsin communities. The Marshfield Clinic Breast Care Program is committed to breast health throughout the continuum from breast cancer risk assessment and prevention, advanced diagnostics, and screening tools to genetic testing and state-of-the-art surgical techniques. In order to provide the highest level of care, we coordinate a breast care team that involves collaboration of several multidisciplinary healthcare professionals. Experts from various departments, including radiologists, pathologists, breast surgeons, medical and radiation oncologists, genetic counselors, clinical trial specialists, and our breast care navigator, all work together to provide cutting edge cancer treatment and management. Our distinctive breast care program allows patients to see multiple providers without having to make multiple appointments and promotes discussion of treatment recommendations and creation of a personalized treatment plan for each patient by a team of specialists. Here we describe the development and establishment of a breast care program with service for rural breast cancer patients.

\section{Rationale for the Development of a Breast Care Program}

Breast cancer continues to be the leading cancer among women in the United States. It is estimated that 232,670 new cases of invasive breast cancer will be diagnosed among women in the United States in 2014, in addition to 62,570 new cases of non-invasive breast cancer. ${ }^{1}$ In 2014 , approximately 40,000 women in the United States will die 


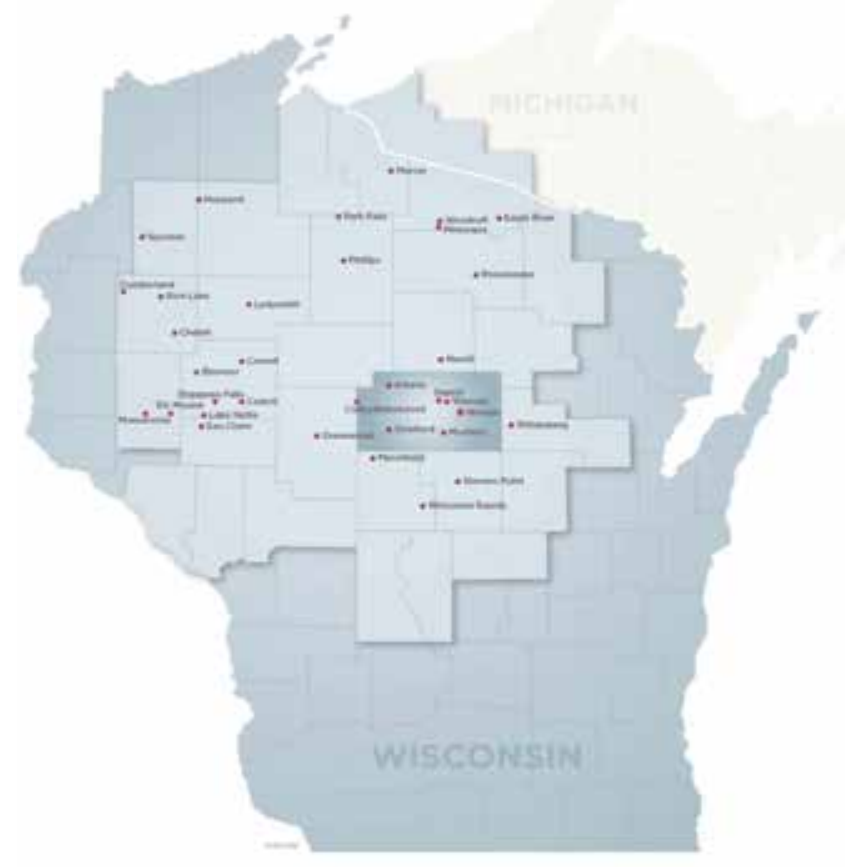

Figure 1: Map of Marshfield Clinic service area with Marathon County highlighted. Seven Marshfield Clinic locations serve the residents of Marathon County, including the Breast Care Program (starred).

from breast cancer, making it the second leading cause of cancer death in women. ${ }^{1}$ Even so, breast cancer mortality has gradually declined since 1989 , especially among younger women. ${ }^{1}$ Increasing survival rates and improved outcomes for women with breast cancer can be attributed to advances in diagnosis and treatment, development of specialized cancer treatment centers, and collaboration by multidisciplinary teams of experts. ${ }^{2}$ It is estimated that there are nearly 2.8 million breast cancer survivors currently living in the United States. ${ }^{3}$

Health care organizations have a vested interest in improving patient outcomes. Prompt and efficient diagnosis and treatment for patients not only decreases patient morbidity and mortality, but also contributes to the provision of cost-effective and timely services with improved patient satisfaction. ${ }^{4}$ Patient care providers have embraced programs that provide breast cancer patients with comprehensive services, improve collaboration among health care professionals, deliver efficient patient-centered care, and identify and address gaps in service.

In the United States, women residing in rural areas face significant challenges related to breast cancer care, largely due to the fact that rural women are often diagnosed with breast cancer at a later stage than women residing in urban areas. ${ }^{5}$ Mammography screening rates are consistently lower among rural women ${ }^{6}$ for a number of reasons, including lack of insurance, geographic distance to screening mammography services, lack of knowledge regarding health issues, lower income, and lower rates of education. ${ }^{7}$ The rural population served by the Marshfield Clinic Health System is no exception. ${ }^{8}$
The Marshfield Clinic Breast Care Program (BCP)-Eastern Division serves the over 130,000 residents of rural Marathon County, Wisconsin (figure 1). Many residents indicate that they do not have appropriate access to health care, and approximately $28 \%$ of families have some level of medical debt. ${ }^{9}$ Within Marathon County, more than $20 \%$ of patients seen by Marshfield Clinic are considered underserved.

The $\mathrm{BCP}$ was developed to provide the rural women residing in Marathon and the surrounding counties with a service delivery structure for coordinated planning and management across a patient's breast cancer experience. By partnering with and navigating patients through this system, the $\mathrm{BCP}$ aims to improve quality, reduce cost, and eliminate barriers to care, while also ensuring timely delivery of services for breast cancer patients. It has been shown that specialized breast cancer care is associated with a significant reduction in mortality ${ }^{4}$ and that a mutual and personal patient-provider relationship is vital to improving outcomes of all aspects of breast cancer care for rural women. ${ }^{2}$ The BCP supports the delivery of breast cancer care through the development and application of a breast cancer patient management framework based on six key principles, including: (1) enhanced access to

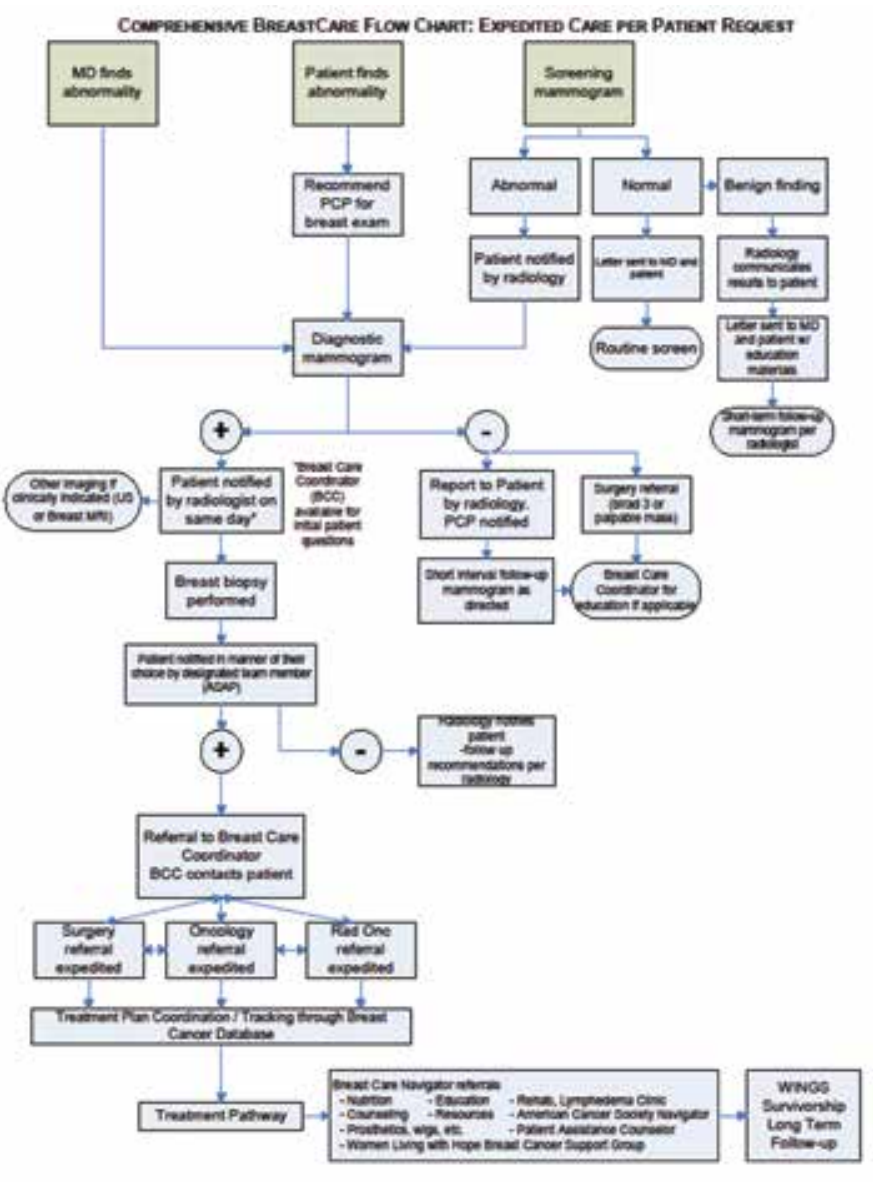

Figure 2: Comprehensive breast care flow chart developed to expedite care following breast cancer diagnosis and adopted by the Marshfield Clinic Breast Care Program. 
Table 1. Services provided by different types of breast centers.

\begin{tabular}{lccccc}
\hline & \multicolumn{5}{c}{ Breast Center Type } \\
\cline { 2 - 5 } Service Component & Screening & Diagnostic & Clinical & Treatment & Comprehensive \\
\hline Outreach-Education & + & + & + & + & + \\
Imaging & + & + & + & + & + \\
Needle Biopsy & - & + & + & + & + \\
Pathology & - & + & + & + \\
Surgical Care & - & - & + & + \\
Plastic Surgery & - & - & + & + \\
Radiation Therapy & - & - & + & + \\
Medical Oncology & - & - & + & + \\
Rehabilitation & - & - & \pm & + \\
High Risk Clinic & - & - & + & + \\
Research & \pm & \pm & +
\end{tabular}

+ , component must be provided by this type of center; \pm , component may be provided, but is optional for this type of center; -, component not expected by this type of center.

services and quality care for all breast cancer patients ranging from prevention, early detection, diagnosis, and treatment through supportive and palliative care; (2) better coordination of high quality, compassionate care with delivery in a multidisciplinary setting; (3) reduction of barriers that prevent timely care, such as transportation, insurance, communication, and financial concerns; (4) improvement of breast cancer patient outcomes and satisfaction; (5) efficient delivery of care that could result in cost savings for the health care system; and (6) enhanced relationships with the community.

\section{Development of the Marshfield Clinic Rural Breast Care Program}

The Marshfield Clinic BCP was established in 2007 when it became evident that newly diagnosed breast cancer patients experienced issues related to access, delays in the trajectory of care, and outflow to external health care organizations. A timeline was initially developed to accommodate care for a breast cancer patient seeking a second opinion for her disease at Marshfield Clinic prompting a local medical oncologist (AAO) and oncology-certified nurse practitioner (JLD) to investigate steps toward development of an accredited breast program. After observing multiple delays in the timeline from diagnosis to treatment, we began to review similar timelines for other rural women diagnosed with breast cancer within Marshfield Clinic Eastern Division.

Areas for improvement in breast care were identified. One of the first improvements made included obtaining commitment from all practicing providers caring for patients in the $\mathrm{BCP}$ to coordinate same day consultations for newly diagnosed women. Another measure for improvement was appointment of a Patient Access Coordinator (PAC). The PAC is responsible for assessment of program eligibility for women who are uninsured or underinsured and assistance with prior authorization for treatment. A Breast Care Coordinator/ Navigator (BCN) was also hired to meet identified needs and guide patients through their breast care journey by assisting with resources, transportation, emotional support, and other arrangements. Implementation of these programs appears to have lessened hardships for many women in the program.

A multidisciplinary team was assembled, including providers from primary care, radiology, surgery, pathology, radiation oncology, oncology, and nursing in addition to administrative personnel. A multidisciplinary breast care pathway/flowchart was created with input from all providers (figure 2). A comprehensive database was created to help follow women diagnosed with invasive and non-invasive breast cancer through the diagnosis and treatment process. A Breast Care Program Leadership Council (BPL) with representatives from administration, nursing, the $\mathrm{BCN}$, and physicians in oncology, radiology, and surgery was assembled.

Acontinuing medical education(CME)-based, multidisciplinary Breast Care Conference was established to assist providers practicing within the $\mathrm{BCP}$ through detailed planning, discussion, and coordination of care for all rural women diagnosed with invasive and non-invasive breast cancer. The Breast Care Conference contributes to enhancing providers' educational requirements regarding the delivery of a coordinated breast cancer care approach. Evidence-based, practice-relevant articles in breast care and national breast care standards are discussed at this conference elevating the participants' level of understanding and expertise in the delivery of breast care services. After constructing the cornerstones of a successful $\mathrm{BCP}$, national certification was accomplished through the National Accreditation Program for Breast Centers (NAPBC) in 2010 and subsequent recertification was achieved in 2013.

\section{Key Components of a Breast Center}

The National Consortium of Breast Centers ( $\mathrm{NCoBC}$ ) recognizes screening, diagnosis, and treatment as key components of breast cancer treatment. ${ }^{10}$ Treatment must 


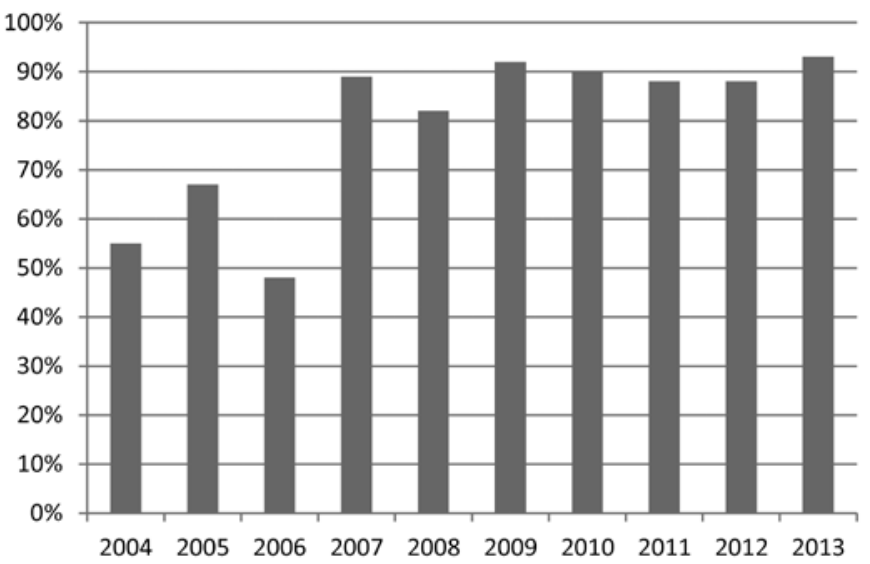

Figure 3: Patient retention before and after establishment of the Breast Care Program in 2007 and through accreditation from National Accreditation Program for Breast Centers in 2010 and reaccreditation in 2013.

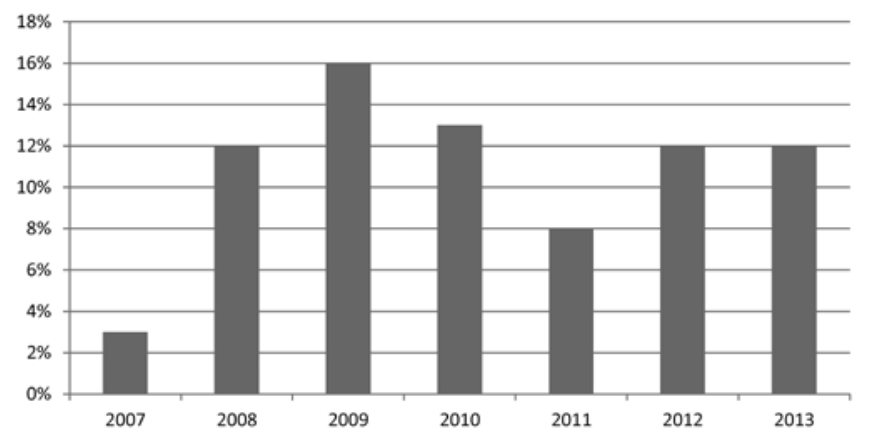

Figure 4: Percent enrollment of breast cancer patients in clinical trials by year.

involve collaboration between numerous specialists in a variety of departments, such as surgery, medical oncology, radiation oncology, radiology, pathology, plastic surgery, physical therapy, behavioral health, and nursing. ${ }^{10}$ Although many of these services exist in most health care systems, a Breast Center focuses on the efficient integration of these multidisciplinary services and results in a synergy that provides a better quality of care than if these entities worked in isolation. ${ }^{10}$ Delivery of breast care services can range from centralized comprehensive breast centers with multidisciplinary consultations in one location to a decentralized model with practitioners in different cities and even organizations. ${ }^{11}$

The NCoBC defines five distinct types of breast centers based on the services encompassed, including screening, diagnostic, clinical, cancer treatment, and comprehensive cancer treatment centers (table 1). The Marshfield Clinic BCP is accredited as a comprehensive breast cancer treatment center, providing all the services described in table 1 in addition to patient navigation and nursing services, regular interdisciplinary breast cancer conferences, data management, quality improvement processes, and a survivorship program.

\section{The Importance of Accreditation in Breast Care}

Recognizing the demand for a program to evaluate and improve the performance of centers focused on the diagnosis and treatment of breast cancer, the American College of Surgeons compiled a group of leaders from professional organizations representing the major disciplines in breast care, eventually resulting in the NAPBC. ${ }^{12,13}$ The mission of NAPBC is to improve the quality of breast care through monitoring the outcomes of patients with breast disease. ${ }^{12}$ Specific NAPBC objectives include development of criteria for breast centers with a survey process to monitor compliance, strengthening of the scientific basis for improving quality care, establishment of a National Breast Disease Database to report patterns of care and improve quality, and reducing breast cancer morbidity and mortality by improving screening mammography and advocating for increased access and participation in clinical trials. ${ }^{12}$

NAPBC accreditation is awarded to centers that willingly pledge to provide the best in breast cancer diagnosis and treatment and comply with established NAPBC standards. ${ }^{12}$ Each center must undergo a thorough appraisal and review of its performance and compliance with the outlined NAPBC standards. ${ }^{12}$ The 27 established NAPBC standards for breast center accreditation encompass six major areas, including (1) center leadership, (2) clinical management, (3) research, (4) community outreach, (5) professional education, and (6) quality improvement. Marshfield Clinic BCP achieved a perfect score for all 27 standards upon both initial accreditation in 2010 and subsequent reaccreditation in 2013.

Accreditation through NAPBC offers several benefits that enhance a breast center and its quality of patient care. ${ }^{12}$ NAPBC-accredited centers offer coordinated multidisciplinary care, commitment to high quality care, national recognition and public promotion of their program, improved patient retention, enhanced timeliness of care, adherence to national guidelines, increased access to and enrollment in research, enriched outreach in the community, and cost savings to their overall program. ${ }^{12}$

\section{Highlights of the Marshfield Clinic Breast Care Program}

Patient Navigation

The Marshfield Clinic BCP BCN role was initiated and designed to: advocate for patients; coordinate patient care; increase and facilitate communication between patients, providers, and family members; reduce disparities in cancer treatment; empower patients with education and information; and assist with identifying community resources, such as financial aid, transportation, and emotional support, for patients and their families. The $\mathrm{BCN}$ also supports the assessment of programs goals and implements changes identified by the Breast Center Leadership. The BCN is employed full-time and achieved certification through $\mathrm{NCoBC}$ as a Certified Breast Patient Navigator in imaging and cancer (CBPN-IC) and through the Oncology Nursing 
Society as a Certified Breast Care Nurse (CBCN). The BCN carries a portable phone and utilizes a laptop computer to facilitate immediate access at various campuses for patients, families, and providers using the electronic medical record.

Consistent with a previous study of breast cancer patient navigation in a rural setting, ${ }^{14}$ the organizational structure of our BCP has led to increased satisfaction for the patient and provider especially due to the characteristic real time accessibility. Within the $\mathrm{BCP}$, the navigator role has been instrumental in improving outcomes-related timeliness of patient care. Following diagnosis, the $\mathrm{BCN}$ offers patients coordinated appointments with oncology, surgery, and radiation oncology. This coordination has ultimately led to increased retention, efficiency, fewer trips to the clinic, and increased patient satisfaction. The navigator then follows the patient throughout their journey with breast cancer into survivorship to meet the goals of the program.

\section{Community Involvement}

In addition to direct patient care, the $\mathrm{BCN}$ coordinates and facilitates the Women Living with Hope breast cancer support group. The group is open to any woman who has a personal experience with breast cancer, regardless of when and where they were diagnosed and treated. The mission of the support group is to provide women in the community affected by breast cancer with informational, emotional, and practical support in a comfortable setting. The group meets monthly and participates in many activities to gain support from one another and to interact with the community. Community partnerships have been established with a local stamp shop to create greeting cards and invitations for new members and a local yoga studio to practice therapeutic yoga for healing of mind, body, and spirit. Support group members also give back by crafting shawls for newly diagnosed breast cancer patients and "recovery buddy bags" for patients actively undergoing treatment.

The Marshfield Clinic BCP also participates in community outreach events to reach rural and underserved women and provide information on breast cancer screening and prevention. With outreach, rural women in the community are allowed to ask questions and receive information regarding breast concerns, breast diseases, and the prevention and treatment services available. Education, networking, and advertising have been provided through presentations to health classes at the University of Wisconsin-Marathon County campus, North Central Technical College, and area Hmong Health Events. In 2013, a unique local community event included a skit-based evening fundraiser called Hope's Chest designed to appeal to both women and men interested in learning more about breast cancer in a fun and entertaining manner.

Comprehensive Breast Care Flow Chart

A Comprehensive Breast Care Flow Chart (figure 2) was established through a multi-disciplinary effort with stakeholder input from the oncology, surgery, radiology, primary care, and obstetrics and gynecology departments as well as management representation from the Marshfield Clinic Eastern Division. The flow chart represents two distinct pathways toward diagnosis depending on whether the patient undergoes diagnostic work-up as a result of clinical symptoms or screening mammogram findings. The pathways merge at the point of diagnostic mammography. The Comprehensive Breast Care Flow Chart succinctly defines and communicates the key clinical and process quality indicators. Guidelines alone were insufficient to promote the new process flow, but promotion of the flow chart by the multidisciplinary team allowed for policies to be transformed into practice with considerable success. Several major changes had to occur to move this process forward. For example, before development of the Comprehensive Breast Care Flow Chart, a significant number of breast biopsies were performed by general surgery without radiology guidance. Changing this practice to include biopsy with radiology guidance was politically charged, but agreed upon by all stakeholders to be in the best interest of the patient. An additional major change was the transition to horizontal consultation of breast specialty services. Before the flow chart was designed, consultations started with surgery followed by oncology and radiation oncology. With the advent of neoadjuvant chemotherapy, a horizontal consultation process lended to improved options for patients and higher collaboration among specialists. A newly diagnosed breast cancer patient is now seen by medical oncology, surgery, and radiation oncology services on the same day, if possible. This allows rural women, who must travel a distance, to more efficiently seek healthcare. The engagement and collaboration of all stakeholders from both primary and specialty care and consideration of the patient perspective in the development of these guidelines has been essential to producing a truly coordinated diagnostic pathway.

\section{Patient Retention}

The BCP was initiated in 2007 and was associated with increased patient retention following breast cancer diagnosis within the Marshfield Clinic Health System (figure 3). We attribute improved patient satisfaction and retention to the seamless flow of care for diagnosis and treatment of breast cancer created by the BCP. As evident in figure 3, following the increase in retention associated with BCP establishment in 2007, stability was observed in association with NAPBC accreditation in 2010. The infrastructural support of the PAC and $\mathrm{BCN}$ is thought to have been paramount to retention of rural breast care patients due to the high level of support and involvement provided throughout the patient's journey.

\section{Clinical Research \\ Marshfield Clinic Research Foundation (MCRF) offers clinical trials for the various stages of breast cancer, including adjuvant, neoadjuvant, metastatic disease, cancer symptom management, and preventative trials. MCRF oncology research nurses screen all newly diagnosed breast cancer}


patients within our breast care program and assist physicians in determining any open clinical trials for which patients may be eligible. The oncology research nurse communicates eligibility and will assist with clinical trial enrollment and study activities. The close relationship between physicians, research staff, oncology nurses, and patients enhances overall satisfaction, participation, and adherence to the clinical plan of care. MCRF participates in several cooperative group studies through the National Cancer Institute Community Oncology Research Program (NCORP), formerly known as Community Clinical Oncology Program (CCOP), in addition to many other industry- and investigator-sponsored trials in which patients can enroll.

Clinical trial enrollment rates are shown in figure 4. In 2013, 4 of 57 patients diagnosed with breast cancer were placed on clinical trials for an overall accrual rate of $12 \%$, which greatly exceeds the NAPBC criteria requirement for accreditation of $2 \%$. Trends in clinical trial participation over time suggest that the BCP allows women to participate in clinical trials despite rural residence.

Breast Care Conference and Continuing Medical Education (CME) Our Interdisciplinary Breast Care Conference is a regularly scheduled conference that takes place twice per month and is approved through the CME Committee at Marshfield Clinic in compliance with Accreditation Council for Continuing Medical Education(ACCME) standards. All areas of the multidisciplinary team are represented, including oncology, surgery, radiology, pathology, plastic surgery, radiation oncology, research, genetics, nurses, nurse practitioners, physical therapists, management, primary care providers, patient assistance counselors, outreach coordinators, and other support staff. Cases are presented via PowerPoint and are video conferenced to outreach areas offsite in our system. All newly diagnosed breast cancer patients in whom treatment has not been initiated or require further discussion are presented to the multidisciplinary group. NAPBC requires that $85 \%$ of these patients are presented prospectively. Other breast cancer patients are presented retrospectively at the request of a provider, and a formal, thorough review is performed where imaging, pathology, National Comprehensive Cancer Network (NCCN) guidelines, and clinical trials are reviewed within the discussion of the overall treatment plan and goals.

\section{WINGS Survivorship Program}

Marshfield Clinic WINGS (We Inspire, Nurture and Give Support) is a cancer survivorship program that offers holistic care for adult patients throughout the cancer care continuum. Cancer affects both patients and their loved ones. ${ }^{15}$ The goal of WINGS is to provide an environment that nurtures healthy survivorship for individuals with cancer experiences. WINGS offers education and resources designed to maximize medical care and quality of life from diagnosis and beyond through an array of tailor-made programs and services for individuals affected by cancer. Breast cancer patients are offered consultation with a survivorship coordinator/registered nurse and encouraged to participate in this program. WINGS utilizes a written cancer treatment summary and follow-up care plan for cancer survivors.

A one-day inclusive survivorship workshop entitled Wellness Beyond Breast Cancer is offered to women post-breast cancer treatment. The workshop focuses on survivorship issues, stress management, and resiliency training. Participants receive a workbook, resource materials, lunch, refreshments, and a graduation gift. The workshop is made available through local grant support, and we are continuing to add new classes in other rural counties to better serve the needs of rural women who reside there.

\section{High Risk Breast Service}

The High Risk Breast Clinic was established as part of the BCP to provide a comprehensive assessment of approximate individual risk for developing breast cancer. Various tools developed to assess personal risk are used to help a patient better understand if her family history fits the pattern of hereditary breast cancer, and genetic testing is reviewed in an individual context. Along with approximation of risk, what is known and unknown about breast cancer risk is discussed. The risk of developing breast cancer is often inaccurate and the High Risk Breast Clinic aims to increase understanding of risk, which can allow an individual to be proactive in incorporating prevention and early detection strategies into their life.

Table 2. American Cancer Society (ACS) benchmark performance.

\begin{tabular}{|c|c|c|c|}
\hline Benchmark & ACS & Broward Health ${ }^{16}$ & Marshfield Clinic \\
\hline Navigation from abnormal mammogram to care & 53 days & 51 days & 23 days \\
\hline Time from abnormal findings to diagnosis & 13 days & 13 days & 7 days \\
\hline Contacted by navigator case manager & 3 days & 1 day & Same day \\
\hline Follow-up procedure scheduled & 4 days & $\leq 1$ day & Same day \\
\hline Referral to cancer center & 7 days & 13 days & 4 days \\
\hline Receive first cancer treatment & 14 days & 12 days & 6 days \\
\hline
\end{tabular}




\section{Outcome Measures}

Continuous evaluation of the Marshfield Clinic BCP using outcome measures developed by the American Cancer Society (ACS) has been in place since program inception in 2007. In table 2, we compare our findings to ACS benchmarks and results recently published by Broward Health, a community health system serving residents of southeastern Florida. Patients and payers seek accredited programs that include documented outcomes that meet or exceed national benchmarks. Tracking of outcome measures suggests that the Marshfield Clinic BCP meets ACS benchmarks and performs on par with larger systems, such as Broward Health in Florida, even in a much smaller, more rural setting.

\section{Conclusion}

Adoption of the navigation flow chart has encouraged timeliness from abnormal mammogram to first treatment and resulted in an efficient breast care program. Patient satisfaction surveys routinely demonstrate a benefit to providing breast cancer services to women through the coordinated Marshfield Clinic BCP. Coordination of services limits the potential for replication and places focus on improving the quality and convenience of care that patients receive. The BCP provides an integrated healthcare approach, promotes clinicianclinician and clinician-patient communication, and supports the institutional goal of delivering the highest-quality care possible. Ultimately, the Marshfield Clinic Breast Care Program-Eastern Division offers rural women access to high quality, expert breast care close to their home and demonstrates that successful breast programs can thrive in rural settings.

\section{Acknowledgements}

The authors thank the Marshfield Clinic Research Foundation for providing assistance in the preparation of this manuscript through the services of the Office of Scientific Writing and Publications.

\section{References}

1. American Cancer Society. Cancer Facts \& Figures 2014. Atlanta: American Cancer Society; 2014. Available at: www.cancer. org/acs/groups/content/@research/documents/webcontent/ acspc-042151.pdf. Accessed November 17, 2014.

2. Rayman KM, Edwards J. Rural primary care providers' perceptions of their role in the breast cancer care continuum. J Rural Health. 2010;26(2):189-195.

3. American Cancer Society. Cancer Facts \& Figures 2013. Atlanta: American Cancer Society; 2013: Available at: www.cancer.org/ acs/groups/content/@epidemiologysurveilance/documents/ document/acspc-036845.pdf. Accessed November 17, 2014

4. Rosselli Del Turco M, Ponti A, Bick U, et al. Quality indicators in breast cancer care. Eur J Cancer. 2010;46(13):2344-2356.

5. Amey CH, Miller MK, Albrecht SL. The role of race and residence in determining stage at diagnosis of breast cancer. $\mathrm{J}$ Rural Health. 1997;13(2):99-108.

6. Engelman KK, Ellerbeck EF, Perpich D, Nazir N, McCarter K, Ahluwalia JS. Office systems and their influence on mammography use in rural and urban primary care. J Rural Health. 2004;20(1):36-42.
7. Lipsky MS, Koenigs M, Nora R, Peralta E, Zahasky K. Breast cancer prevention for rural healthcare practitioners. Am J Nurse Pract. 2008;12:49-58.

8. Onitilo AA, Engel JM, Liang H, et al. Mammography utilization: patient characteristics and breast cancer stage at diagnosis. AJR Am J Roentgenol. 2013;201(5):1057-1063.

9. Marathon County Wisconsin. Marathon County Wisconsin Central Time: About Marathon County. Available at: www. co.marathon.wi.us. Accessed November 17, 2014.

10. National Consortium of Breast Centers. Breast Center Types and Criteria. Available at: www.breastcare.org. [Membershiponly site.] Accessed November 17, 2014.

11. Bickell NA, Young GJ. Coordination of care for early-stage breast cancer patients. J Gen Intern Med. 2001;16(11):737-742.

12. American College of Surgeons. National Accreditation Program for Breast Centers (NAPBC) Standards. Available at: napbc-breast. org/standards/standards.html. Accessed November 17, 2014.

13. Winchester DP. Evaluating and managing breast disease in the United States: Are we properly organized? J Surg Oncol. 2010;102(4):299-300.

14. Hook A, Ware L, Siler B, Packard A. Breast cancer navigation and patient satisfaction: exploring a community-based patient navigation model in a rural setting. Oncol Nurs Forum. 2012;39(4):379-385.

15. Marshfield Clinic WINGS Cancer Survivorship Services. Available at: www.marshfieldclinic.org/specialties/cancercare/wings-survivorship-home/wings-survivorship-services. Accessed November 17, 2014.

16. Delvaille P. Broward Health's breast cancer navigation program: Meeting the needs of underserved patients. ACCC's Cancer Care Patient Navigation: A Call to Action. Available at: http://www.browardhealth.org/upload/docs/navigator program_article.pdf. Accessed November 17, 2014.

\section{Author Affiliations}

Jill L. Depke, MSN, APNP, AOCNP, $C B C N^{*}+$; Amanda Boreen, $R N, C B P N-I C, C B C N^{*}$; and Adedayo A. Onitilo, $M D, P h D$, $M S C R, F A C P^{*}$

*Marshfield Clinic-Weston Center, Cancer Care-Hematology Oncology, Weston, Wisconsin, USA

${ }^{\dagger}$ Current Affiliation: Medical College of Wisconsin, Clinical Oncology, Milwaukee, Wisconsin, USA 